\title{
Multimodal Automatic Indexing for Broadcast Soccer Video
}

\author{
Naoki Uegaki, Masao Izumi, and Kunio Fukunaga \\ Osaka Prefecture University, Sakai, Osaka 599-8531, Japan \\ izumi@cs.osakafu-u.ac.jp, \\ http://www.com.cs.osakafu-u.ac.jp/
}

\begin{abstract}
In this paper, we propose a novel method for estimating major soccer scenes from cameraworks and players trajectories based on probabilistic inference, and annotating scene indexes to broadcast soccer videos automatically. In our method, we define relations between cameraworks and scenes, and between players trajectories and scenes by conditional probabilities. Moreover defining temporal relations of scenes by transition probabilities, we represent those relations as dynamic bayesian networks (DBNs). And those probabilities are evaluated by learning parameters of the networks. After extracting the cameraworks and the players trajectories, we compute the posterior probability distribution of scenes, and give the computed results to the soccer video as the scene index. Finally, we discuss the extendibility of the proposal indexing technique in the case of adding ball trajectories and audios.
\end{abstract}

\section{Introduction}

In recent years, the spread of cable TV, DVD recorders, etc, enabled individuals to record a lot of TV programs easily. But, it needs immense time and efforts to search scenes that you want to watch in large amount of videos. Then, the technology which gives an effective index automatically will be more indispensable from now on.

However, it is difficult to create a general technique of indexing for all kinds of video. On the other hand, domain knowledges which specialized in the specific kind of video are effective information for indexing. Using domain knowledges, there are many researches about scene estimation, event estimation, and indexing. Leonardi at al. [1] estimate major soccer scenes using cameraworks peculiar to soccer videos, for example it pans and zooms rapidly on shoot scenes and corner kick scenes. Xinghua et al. [2] estimate goal events using textures and score boards peculiar to soccer videos. If you use domain knowledges, it becomes easy to indexing. Moreover, it takes into consideration that a video is generally multiple streams of media information, such as audios, texts, and images, it will be thought that the performance of indexing can be raised more by unifying multimodal information. However, unifying multimodal information, we must be careful of there being merit and demerit in each information. For example 
about audios, if words pronounced by the announcer are extracted, it will become an effective information for scene estimation, but word extraction has a high dependence to the announcer. About texts, although it will also become an effective information on scene estimation, having a high dependence to the text creater, and about cameraworks, although a camera performs specific work in each scene, it depends the cameraman. On the other hand, about players and ball trajectories, which are difficult to extract, they don't depend on the announcer and little depend on the cameraman.

In this paper, we propose a novel method for estimating major soccer scenes from cameraworks and players trajectories, and annotating scene indexes to soccer videos automatically. In proposal method, to make the most of the merit of cameraworks and players trajectories, we unify these two informations using bayesian networks. Moreover, for the purpose of considering of temporal transition of scenes, we use dynamic bayesian networks (DBNs) [3],[4] to estimate scenes.

This paper is organized as five sections, the section 2 shows our approach overview for automatic indexing, we discuss scene indexing and retrieval using DBNs in the section 3, the section 4 shows indexing performance to a broadcast soccer video, and the section 5 gives the conclusions.

\section{Our Approach Overview}

\subsection{Shot Classification}

A soccer video can be mainly classified to the following three shots (a shot is an time continuous image sequence by the same camera) [5]. A playing field shot displays the global view of the field. A player shot shows the view of one or a few persons. And an audience shot is denoted as out of the field, mostly audience seats. Applying the method proposed by Uegaki et al. [6], we classify the soccer video to these three shots. Below this process, since the playing field shot includes many important scenes, we foucus only on the playing field shot.

\subsection{Estimation of Projective Transformation Matrix}

It needs projective transformation matrix, which transforms the image coordi-

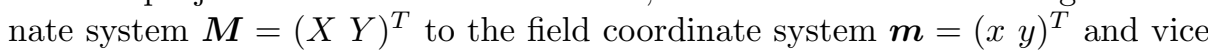
versa, to extract cameraworks and players trajectories. Applying the method proposed by Nakatsuji et al. [7], we estimate projective transformation matrix $P$ (which is $3 \times 3$ matrix) automatically. Then we can transform the image coordinate system and the field coordinate system each other by the equation (1).

$$
\lambda \tilde{\boldsymbol{M}}=P \tilde{\boldsymbol{m}}
$$

Here, $\tilde{\boldsymbol{M}}=\left(\begin{array}{lll}X Y & 1\end{array}\right)^{T}, \tilde{\boldsymbol{m}}=\left(\begin{array}{lll}x y & 1\end{array}\right)^{T}$, and $\lambda$ is a scalar. 


\subsection{Extraction of Cameraworks}

Cameraworks in playing field shots are often performed as the ball position is the center of image, so that the trajectory of the center-of-image projected on the field coordinate system is an important key to estimate scenes. For example, we can observe the trajectory of the center-of-image which goes to the goal area rapidly on shoot scenes. Moreover, the area of the soccer court on the image is an important key, too. For example, we can observe that the area of the soccer court becomes much smaller with fast zoom-in on a corner-kick scene. Accordingly we extract the trajectory of the center-of-image and the area of the soccer court as cameraworks. So we define cameraworks as following vector at frame $f$.

$$
\boldsymbol{c}_{f}=\left(\begin{array}{llllll}
x_{c} & y_{c} & v_{x_{c}} & v_{y_{c}} & a_{c} & v_{a_{c}}
\end{array}\right)
$$

Here, $\boldsymbol{m}_{c}=\left(\begin{array}{ll}x_{c} & y_{c}\end{array}\right)$ and $\boldsymbol{v}_{\boldsymbol{m}_{c}}=\left(v_{x_{c}} v_{y_{c}}\right)$ are the position and velocity of the center-of-image on the field coordinate system. $a_{c}$ and $v_{a_{c}}$ are the area of a soccer court and the velocity of it. This vector is extracted at each frame.

\subsection{Extraction of Players Trajectories}

Applying the method proposed by Uegaki et al. [8], the trajectories of each player on the field coordinate system are extracted. But it is very difficult to get exact trajectory of each player because of frequent overlap of mltiple players in a soccer video. On the other hand, it doesn't always need exact trajectory of each player to estimate scenes. For exsample on shoot scenes, the trajectory of the mean position of players usually goes to the goal area. Accordingly we extract the trajectory of the mean position of players, the variance of the players position, and the number of players as players trajectories. So we define players trajectories as following vector at frame $f$.

$$
\boldsymbol{p}_{f}=\left(\begin{array}{llllll}
x_{p} & y_{p} & v_{x_{p}} & v_{y_{p}} & V_{p} & v_{V_{p}} \\
N_{p}
\end{array}\right)
$$

Here, $\boldsymbol{m}_{p}=\left(x_{p} y_{p}\right)$ and $\boldsymbol{v}_{\boldsymbol{m}_{p}}=\left(v_{x_{p}} v_{y_{p}}\right)$ are the mean position of players and the velocity of it on the field coordinate system. $V_{p}$ and $v_{V_{p}}$ are the variance of the players position and the velocity of it, and $N_{p}$ is the number of players. This vector is extracted at each frame.

\subsection{Scene Indexing and Retrieval}

After extracting the camerawork vector $\boldsymbol{c}_{f}$ and the players trajectory vector $\boldsymbol{p}_{f}$ at each frame, we compute the posterior probability distribution of scenes based on the DBNs (shown in Figure 1), and annotate computed results to the soccer video. Then we retrieve a desirable scene from the indexed video. Next section gives a detailed explanation of it. 


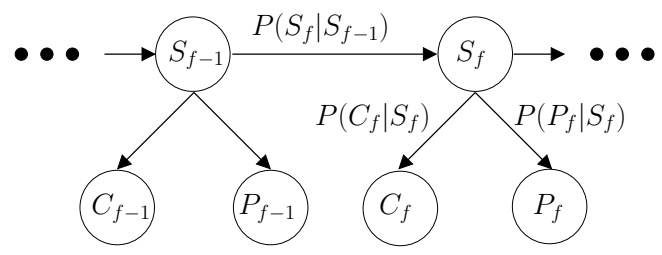

Fig. 1. The DBNs for scene estimation

\section{$3 \quad$ Scene Indexing and Retrieval}

Let us estimate six scenes, those are shoot, corner kick, free kick, throw-in, goal kick, and others. This section gives how to estimate these scenes and retrieve them from the indexed video. Figure 1 shows the DBNs for the purpose of estimating these scenes. Node $S$ called hidden node represents random variables of these scenes, and Table 1 shows the values which node $S$ can take. Node $C$ represents random variables of camerawork vectors, and node $P$ represents random variables of players trajectory vectors. Node $C$ and $P$ are called observation node. After observing (extracting) the camerawork vector $\boldsymbol{c}_{f}$ and the players trajectory vector $\boldsymbol{p}_{f}$ at each frame, we set observation nodes with these observation values, then we compute the posterior probability distribution of scenes and annotate the computed results to the video.

Table 1. Target scenes to estimate

\begin{tabular}{l|l}
\hline Scene name & $S$ \\
\hline \hline shoot & $s^{1}$ \\
\hline corner kick & $s^{2}$ \\
\hline free kick & $s^{3}$ \\
\hline throw-in & $s^{4}$ \\
\hline goal kick & $s^{5}$ \\
\hline others & $s^{6}$ \\
\hline
\end{tabular}

\subsection{Clustering Observation Vectors}

Extracted camerawork vectors and players trajectory vectors tend to be sparse, because several elements of these vectors are plotted on the soccer field and this field is very vast for these vector elements. Then it will be very difficult to learn observation probability parameters of DBNs in the case of using these vectors directly. In order to reduse the number of observation nodes, we use k-means clustering technique to classify these obervation vectors to several classes. If the number of clusters for camerawork vectors is $K_{C}$, camerawork vectors are categolized to $K_{C}$ classes $\left\{\tilde{c}^{1}, \tilde{c}^{2}, \cdots, \tilde{c}^{K_{C}}\right\}$, and if the number of clusters for players trajectory vectors is $K_{P}$, players trajectory vectors are categolized to 
$K_{P}$ classes $\left\{\tilde{p}^{1}, \tilde{p}^{2}, \cdots, \tilde{p}^{K_{P}}\right\}$. After clustering these vectors, we use these classes instead of vectors themselves to learn observation probability parameters.

\subsection{Scene Indexing}

Let us focus on a playing field shot (from frame $f_{1}$ to $f_{2}$ ). Now the observation sequence from frame $f_{1}$ to $f_{2}\left(o_{f_{1}: f_{2}}=\left\{\tilde{c}_{f_{1}: f_{2}}, \tilde{p}_{f_{1}: f_{2}}\right\}\right)$ was set to observation nodes, we are able to compute the posterior probability distribution of scenes $P\left(S_{f} \mid o_{f_{1}: f_{2}}\right)$ by the following equation (4) which is called fixed interval smoothing [4], provided that transition probabilities of scenes $P\left(S_{f} \mid S_{f-1}\right)$, observation probabilities of cameraworks $P\left(C_{f} \mid S_{f}\right)$ and observation probabilities of players trajectories $P\left(P_{f} \mid S_{f}\right)$ are respectively defined. These probabilities are defined by parameter learning.

$$
\begin{aligned}
P\left(S_{f} \mid o_{f_{1}: f_{2}}\right) & =P\left(S_{f} \mid o_{f_{1}: f}, o_{f+1: f_{2}}\right) \\
& =\alpha P\left(S_{f} \mid o_{f_{1}: f}\right) P\left(o_{f+1: f_{2}} \mid S_{f}\right)
\end{aligned}
$$

Here, $\alpha$ is a normalizing constant, and

$$
\begin{aligned}
& P\left(S_{f} \mid o_{f_{1}: f}\right) \\
= & P\left(o_{f} \mid S_{f}\right) \sum_{S_{f-1}} P\left(S_{f} \mid S_{f-1}\right) P\left(S_{f-1} \mid o_{f_{1}: f-1}\right) \\
P & \left(o_{f+1: f_{2}} \mid S_{f}\right) \\
= & \sum_{S_{f+1}} P\left(o_{f+1} \mid S_{f+1}\right) P\left(o_{f+2: f_{2}} \mid S_{f+1}\right) P\left(S_{f+1} \mid S_{f}\right)
\end{aligned}
$$

Here, $P\left(o_{f} \mid S_{f}\right)$ is denoted as follows.

$$
P\left(o_{f} \mid S_{f}\right)=P\left(\tilde{c}_{f} \mid S_{f}\right) P\left(\tilde{p}_{f} \mid S_{f}\right)
$$

Finally, we annotate the computed result to the video.

\subsection{Parameter Learning}

If there are enough training data, we assume that the goal of learning in this case is to find the maximum likelihood estimates (MLEs) of the parameters $\left(P\left(S_{f} \mid S_{f-1}\right), P\left(C_{f} \mid S_{f}\right)\right.$ and $\left.P\left(P_{f} \mid S_{f}\right)\right)$. The log-likelihood of the training set $D=\left\{D_{1}, \cdots, D_{m}\right\}$ is a sum of terms, one for each node [4]:

$$
\begin{aligned}
L & =\sum_{i=1}^{n} \sum_{m=1}^{M} \log P\left(X_{i} \mid \mathbf{P a}\left(X_{i}\right), D_{m}\right) \\
& =\sum_{i j k} N_{i j k} \log \theta_{i j k}
\end{aligned}
$$


where $\mathbf{P a}\left(X_{i}\right)$ are the parents of $X_{i}, N_{i j k}$ is the number of the events $\left(X_{i}=\right.$ $\left.k, \mathbf{P a}\left(X_{i}\right)=j\right)$ which are seen in the training set, and $\theta_{i j k}$ is defined as $\theta_{i j k}=$ $P\left(X_{i}=k \mid \mathbf{P a}\left(X_{i}\right)=j\right)$. The MLEs which maximize the equation (5) is shown in the equation (6).

$$
\hat{\theta}_{i j k}^{M L}=\frac{N_{i j k}}{\sum_{k^{\prime}} N_{i j k^{\prime}}}
$$

However if there are a small number of training cases compared to the number of parameters, the reliability of MLEs decreases. In this case, assuming that any event occurs more than one time, we use the maximum a posteriori (MAP) estimates instead of MLEs. The MAP estimates is shown in the equation (7).

$$
\hat{\theta}_{i j k}^{M A P}=\frac{N_{i j k}+1}{\sum_{k^{\prime}} N_{i j k^{\prime}}+Z}
$$

where $Z$ is the amount of values which $X_{i}$ can take.

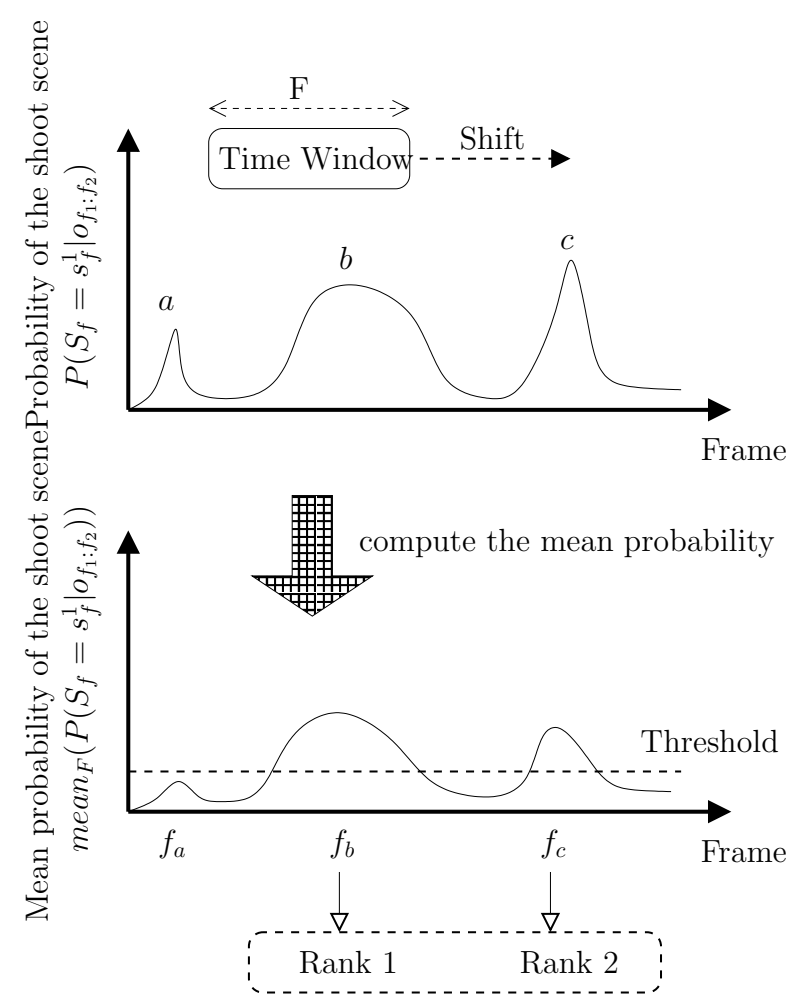

The retrieval results of shoot scenes

Fig. 2. The method of scene retrieval 


\subsection{Scene Retrieval}

Let us think the method of retrieving scenes from a video. After computing the posterior probability distribution of scenes $P\left(S_{f} \mid o_{f_{1}: f_{2}}\right)$, we can get the video with probabilities of scenes. But it has some difficulty to retrieve scenes from this video. For example, suppose that there is a video with probability of the shoot scene as shown in Figure 2. Then the scene around $b$ should be firstly retrieved as a shoot scene because the probability of the shoot scene around $b$ rises most stably compared to around $a$ and $c$. To achieve this, we compute the mean probability $\operatorname{mean}_{F}\left(P\left(S_{f}=s_{f}^{1} \mid o_{f_{1}: f_{2}}\right)\right)$ by shifting the time window which length is $F$ to $P\left(S_{f}=s_{f}^{1} \mid o_{f_{1}: f_{2}}\right)$ and computing the mean probability in the time window as shown in Figure 2. Next we search maximums which are larger than the threshold in $\operatorname{mean}_{F}\left(P\left(S_{f}=s_{f}^{1} \mid o_{f_{1}: f_{2}}\right)\right)$, and if $f_{b}$ and $f_{c}$ are found, since the mean probability at $f_{b}$ is larger than at $f_{c}$, then we present the scene around $f_{b}$ firstly (Rank 1) and the scene around $f_{c}$ secondly (Rank 2 ) as the retrieval result of shoot scenes. The other scenes are retrieved in a similar way.

\section{Experiments and Discussions}

We applied the proposal method to a broadcast soccer video (resolution is $320 \times 240$, frame rate is $30 / s$ ). In this experiment, we prepared a training data of about 18000 frames (about 10 minutes), and after performing the shot classification mentioned in 2.1 , there were 5 shoot scenes, 1 corner kick scene, 0 free kick scene, 6 throw-in scenes, and 2 goal kick scenes in playing field shots. Since there are a small number of training data, observation probabilities were learn using the MAP estimates mentioned in 3.3. On the other hand, transition probabilities was defined manually because the amount of transition probabilities is small (which is $6 \times 6=36$ ). Table 2 shows the transition probabilities, but the observation probabilities are too large to describe in this paper. This time, projective transformation matrix was estimated manually.

Table 2. The transition probabilities $P\left(S_{f} \mid S_{f-1}\right)$

\begin{tabular}{c|c|c|c|c|c|c}
\hline & $s_{f-1}^{1}$ & $s_{f-1}^{2}$ & $s_{f-1}^{3}$ & $s_{f-1}^{4}$ & $s_{f-1}^{5}$ & $s_{f-1}^{6}$ \\
\hline$s_{f}^{1}$ & 0.90 & 0.00 & 0.00 & 0.00 & 0.00 & 0.02 \\
\hline$s_{f}^{2}$ & 0.00 & 0.90 & 0.00 & 0.00 & 0.00 & 0.02 \\
\hline$s_{f}^{3}$ & 0.00 & 0.00 & 0.90 & 0.00 & 0.00 & 0.02 \\
\hline$s_{f}^{4}$ & 0.00 & 0.00 & 0.00 & 0.90 & 0.00 & 0.02 \\
\hline$s_{f}^{5}$ & 0.00 & 0.00 & 0.00 & 0.00 & 0.90 & 0.02 \\
\hline$s_{f}^{6}$ & 0.10 & 0.10 & 0.10 & 0.10 & 0.10 & 0.90 \\
\hline
\end{tabular}

Table 3 shows the retrieval results of scenes against this trainig data. The average of precision rate of the entire retrieval results was $98 \%$, and the recall rate was $100 \%$. 
Table 3. Evaluations of the scene retrieval performance for training data

\begin{tabular}{c|c|c}
\hline Scene name & Precision & Recall \\
\hline shoot & 0.92 & 1.00 \\
corner kick & 1.00 & 1.00 \\
free kick & 1.00 & 1.00 \\
throw-in & 1.00 & 1.00 \\
goal kick & 1.00 & 1.00 \\
\hline average & 0.98 & 1.00 \\
\hline
\end{tabular}

\section{Conclusions}

In this paper, we present a novel framework to indexing from multiple informations. This time, we implemented this framework with cameraworks and players trajectories, and confirmed that major soccer scenes can be estimated moderately for the training data only from these two informations. If ball trajectories or audios are extracted, our framework can be easily extended by adding a ball trajectories node or an audios node to the DBNs mentiond in 3, and we expect more effective results of scene indexing and retrieval. Our future works are to introduce ball trajectories and audios, and to prepare more training and testing data.

\section{References}

1. R. Leonardi, P. Migliorati, "Semantic Indexing of Multimedia Documents," IEEE Multimedia, pp.44-51, April-June 2002.

2. S. Xinghua, J. Guoying, H. Mei, and X. Guangyou, "Bayesian network based soccer video event detection and retrieval, " Proceedings of SPIE - The International Society for Optical Engineering 5286, pp. 71-76, 2003.

3. S. Russell, and P. Norvig, "Artificial Intelligence: A Modern Approach(Second Edition), " Prentice Hall, Chapter 15, pp.537-581. 2002.

4. Kevin P. Murphy, "Dynamic Bayesian Networks: Representation, Inference and Learning, " PhD thesis, University of California, 2002.

5. J. Assfalg, M. Bertini, C. Colombo, and A. Del Bimbo, "Semantic Annotation of Sports Videos," IEEE Multimedia, pp.52-60, April-June 2002.

6. N. Uegaki, K. Nakatsuji, M. Izumi, K. Fukunaga, "Automatic indexing for broadcast soccer video using multiple information," MIRU2004, pp.II-329-334, July 2004. (in Japanese)

7. K. Nakatsuji, N. Uegaki, M. Izumi, K. Fukunaga, "Estimation of Players' Position from Image Sequences of Soccer Game TV Program, " IEICE Technical Report, PRMU2003-214, pp.95-100, Jan. 2004. (in Japanese)

8. N. Uegaki, K. Nakatsuji, M. Izumi, K. Fukunaga, "Tracking of Multiple Players from Soccer Game TV Programs, " IEICE General Conference, no.D-12-112, p.273, Mar. 2003. (in Japanese) 